\title{
La réputation collective sur les marchés agricoles
}

Collective Reputation in Agricultural Markets

\section{Angelo Zago}

\section{(2) OpenEdition}

\section{Journals}

Édition électronique

URL : http://journals.openedition.org/economierurale/4557

DOI : $10.4000 /$ economierurale.4557

ISSN : 2105-2581

Éditeur

Société Française d'Économie Rurale (SFER)

Édition imprimée

Date de publication : 15 février 2015

Pagination : 29-51

ISSN : 0013-0559

Référence électronique

Angelo Zago, "La réputation collective sur les marchés agricoles», Économie rurale [En ligne], 345 | Janvier-février 2015, mis en ligne le 15 février 2017, consulté le 02 mai 2019. URL : http:// journals.openedition.org/economierurale/4557 ; DOI : 10.4000/economierurale.4557 


\section{La réputation collective sur les marchés agricoles}

Angelo ZAGO • Dipartimento di Scienze Economiche, Università degli Studi di Verona, Vérone Italie ; angelo.zago@univr.it

Dans l'agriculture, il existe de nombreux exemples où la coordination est assurée via la réputation collective, par exemple par les indications géographiques, les coopératives de commercialisation et de transformation, I'adhésion à des labels tels que le Label Rouge, etc. L'auteur traite ici de certains des problèmes rencontrés dans ce domaine sur les marchés agricoles, où ces initiatives sont gérées par des entreprises par ailleurs indépendantes, dans le cadre d'un processus de décision démocratique. Après avoir procédé à un examen sélectif de la littérature publiée sur ce sujet, il présente certains des problèmes rencontrés par le secteur et par les autorités et suggère quelques pistes pour les recherches à venir.

MOTS-CLÉS : réputation collective, labels, qualité, $A O C$, vins

\section{Collective Reputation in Agricultural Markets}

In agriculture, there are many instances of coordination obtained via collective reputation, such as geographical indications, marketing and processing cooperatives, membership in labels such as Label Rouge, etc. We discuss some of their problems in agricultural markets, where they are managed by otherwise independent firms through a democratic decision process. After selectively reviewing the published literature, we highlight some relevant problems for the industry and for policy-making and we suggest some venues for future research. (JEL: L22, Q13)

KEYWORDS: collective reputation, labels, quality, PDO, wine

a coordination consiste à réguler divers éléments d'un système de manière à obtenir un fonctionnement intégré et harmonieux. La coordination est donc l'acte d'arranger les choses et de les mettre en ordre ou de les faire fonctionner harmonieusement les unes avec les autres. Dans de nombreux environnements, le système de prix coordonne efficacement les activités économiques. Cependant, il n'est pas rare, par exemple en présence d'un nombre d'acteurs réduit, ou lorsque l'information n'est pas complète, que le système de marché ne fonctionne pas correctement ; d'autres moyens apparaissent alors pour coordonner les activités économiques, comme le montrent d'autres contributions dans ce numéro spécial.

Dans le présent article, nous nous intéressons à un type de coordination particulier, fondé sur la réputation collective, dans lequel des entreprises par ailleurs indépendantes partagent une marque commune (régionale, par exemple). Cela fait longtemps que la réputation est reconnue comme une force du marché propice à l'exécution des contrats (Klein et Leffler, 1981). Lorsque l'information sur certaines caractéristiques ou certains comportements des parties prenantes à une transaction fait défaut, les transactions répétées et l'émergence d'une réputation peuvent corriger les dysfonctionnements du marché qui se produisent à l'occasion des interactions non récurrentes. Voilà longtemps que l'on étudie la réputation individuelle, c'est-à-dire la réputation qui se forme sur la base du comportement des agents individuels, et la littérature sur ce sujet est aujourd'hui assez abondante (pour 
une étude introductive, voir, par exemple, Bar-Isaac et Tadelis, 2008). En revanche, la littérature sur la réputation collective est dans l'ensemble plutôt restreinte (pour une contribution qui a fait date, voir Tirole, 1996), et elle est encore plus rare s'agissant du secteur agricole, alors qu'il existe de nombreux exemples de partage d'une marque commune par des producteurs agricoles.

En Europe, les exemples de réputation collective probablement les plus connus dans le secteur agricole sont les vins d'appellation d'origine protégée (AOP) (autrefois dits d'appellation d'origine contrôlée, ou AOC) ${ }^{1}$. Les AOP, ainsi que les indications géographiques protégées (IGP), constituent la colonne vertébrale du système d'indications géographiques (IG), par lequel les autorités de réglementation de l'Union européenne (UE) ont institutionnalisé le lien entre les attributs qualitatifs d'un bien et le territoire géographique sur lequel il est produit, c'est-à-dire le terroir. Les vins de Bordeaux, de Champagne et de Chianti en constituent des illustrations types : les vignerons de ces régions peuvent vendre leur vin, à condition qu'il soit produit dans le respect de normes communes (appelées le cahier des charges), en utilisant le nom de la région pour le rendre plus facilement reconnaissable, et éventuellement pour bénéficier d'une meilleure réputation.

Les vins AOP sont peut-être les exemples les plus connus, mais il en existe beaucoup d'autres dans l'industrie agroalimentaire, par exemple les fromages Camembert ou Parmiggiano Reggiano, le jambon de Parme ou le Somerset Cider Brandy (eau-de-vie de cidre produite traditionnellement dans le comté de Somerset,

1. Depuis l'adoption du Règlement (CE) $n^{\circ} 479 / 2008$, tous les États membres de l'Union européenne ont harmonisé les règles régissant les AOC pour le vin, et ces vins sont aujourd'hui dits « d'appellation d'origine protégée » (AOP). en Angleterre), etc. On a retrouvé des traces indiquant que l'on associait des produits agricoles de grande qualité provenant de zones spécifiques à des noms géographiques dès le quatrième siècle avant J.-C. (Bertozzi, 1995). Grâce à ces marques régionales, les produits agricoles sont plus aisément reconnaissables par le consommateur et peuvent bien souvent être vendus plus cher, ce qui procure un meilleur profit aux entreprises. C'est la raison pour laquelle le public apprécie et soutient ces efforts collectifs déployés par les entreprises agricoles dans de nombreux pays ${ }^{2}$.

Mais la réputation collective ne se borne pas aux marques régionales. Les adhérents qui livrent leur lait pour la transformation dans une coopérative partagent aussi, dans une certaine mesure, une réputation collective. L'affiliation au Label Rouge constitue un autre exemple. Toutes ces entreprises bénéficient d'une réputation partagée, mais sont également exposées aux risques résultant des actions engagées par les autres entreprises associées à la marque collective, et qui peuvent in fine porter atteinte à leur bien-être économique.

Le présent article a deux objectifs. Premièrement, nous analysons le fonctionnement des marques collectives, en particulier les interactions entre les entreprises qui les créent et les gèrent, et nous mettons en évidence certains des problèmes inhérents à la réputation collective sur les marchés agricoles. La législation accorde en général à un groupe d'entreprises (ou de producteurs agricoles) le pouvoir de gérer

2. Au niveau de l'UE, voir le Règlement initial $\mathrm{n}^{\circ}$ 2081/1992 et ses modifications successives (la dernière en date est le Règlement (CE) sur les appellations d'origine protégées (AOP) et les indications géographiques protégées (IGP). En 2010, l'UE comptait 872 produits agricoles protégés par des indications géographiques (vins non compris), représentant un chiffre d'affaires total de près de 16 milliards d'euros (http://ec.europa.eu/agriculture/quality/schemes/index_en.htm). 
le fonctionnement de leur marque collective : le groupe peut décider qui sera autorisé à s'y affilier et qui devrait être exclu en cas de violation, et il peut définir les règles de production, les contrôles et les sanctions éventuelles, etc.

Ainsi, l'AOP est détenue et gérée par le groupe selon un principe démocratique. Cependant, il arrive qu'au sein d'une marque collective, les entreprises soient hétérogènes, et donc sensibles à des incitations différentes, et qu'elles ne retirent pas le même bénéfice des opportunités susceptibles de découler des efforts collectifs. Profiter de ces opportunités de marché entraîne souvent des choix qui peuvent avoir des impacts différenciés sur les membres, si bien que certains seront désireux de saisir ces opportunités tandis que d'autres s'y refuseront, craignant peut-être que ces choix ne compromettent la réputation du groupe. Nous allons illustrer certains de ces litiges récents entre membres et les choix controversés qui en ont résulté, opérés soit par le groupe gérant l'AOP, soit par certains membres en désaccord avec les décisions de la majorité. Quelques-uns de ces choix controversés sont même parvenus jusqu'aux médias, qui se sont fait l'écho des positions et des intérêts divergents au sein de ces groupes.

Nous nous intéresserons aux problèmes rencontrés par la filière et nous décrirons plusieurs cas récents, mais nous nous pencherons également sur certaines des questions auxquelles doivent répondre les autorités dans ce domaine, en particulier sur celle de la structure hiérarchique ou de gouvernance dont il faudrait doter le système de réputation collective, par exemple une indication géographique, pour éviter ou limiter les litiges entre intérêts divergents, et éventuellement pour faire concorder ces intérêts. Nous comparerons donc le cas de différentes AOP en soulignant certaines des questions qui se posent et qui mériteraient d'être explorées plus avant.
Le deuxième objectif de cet article est de suggérer des pistes de recherche qui pourraient contribuer à la formulation de politiques plus adaptées aux systèmes de réputation collective. Après avoir rapidement passé en revue la littérature sur la réputation individuelle $^{3}$, nous analyserons la réputation collective en nous appuyant sur les évolutions récentes de l'économie de la réputation. La littérature abondante et de plus en plus fournie consacrée à cette question peut être grossièrement et arbitrairement subdivisée en deux types d'ouvrages : ceux qui traitent des modèles d'imitation (lorsque les acteurs de qualité inférieure souhaiteraient être confondus avec les acteurs de bonne qualité) et ceux qui se penchent sur les modèles de séparation (lorsque les acteurs de bonne qualité ne veulent pas être confondus avec les acteurs de qualité inférieure [Bar-Isaac et Tadelis, 2008]).

Nous analyserons certains aspects des problèmes qui sont apparus lors des récentes controverses impliquant des entreprises affiliées à des marques collectives, et nous décrivons d'autres cas présentant les caractéristiques que nous venons d'exposer. Nous avancerons que plusieurs des problèmes mis en lumière ici n'ont pas encore fait l'objet d'une analyse poussée, en particulier le processus de décision démocratique et l'hétérogénéité des entreprises. Nous attirerons enfin l'attention sur des questions qui mériteraient d'être approfondies aux fins de l'élaboration de la politique publique, et nous conclurons cet article.

\section{La littérature sur la réputation}

Il existe de nombreuses situations dans lesquelles les consommateurs ne seront

\footnotetext{
3. Dans cet article, nous laissons de côté la littérature sur les indications géographiques, car la plupart de ces ouvrages ne s'appuient pas sur des modèles de réputation. De plus, à une exception près, nous ne travaillons que sur des documents publiés, et non sur des documents de travail.
} 
renseignés sur la qualité d'un bien qu'après l'avoir consommé, comme dans le cas des biens d'expérience (Nelson, 1970), et dans lesquelles l'asymétrie d'information peut se traduire par des dysfonctionnements du marché (Akerlof, 1970). Différentes solutions peuvent apparaître alors sur le marché pour atténuer ces dysfonctionnements, et la réputation est considérée comme un moyen efficace d'y parvenir (Klein et Leffler, 1981). Lorsqu'un consommateur n'est pas en mesure d'apprécier certains des attributs qualitatifs d'un bien ou d'un service avant de le consommer, il peut se fier à la réputation de l'entreprise ${ }^{4}$, c'està-dire à sa croyance sur la qualité que l'on peut associer au produit de cette entreprise.

L'acheteur peut en effet réunir des informations et se forger une croyance à partir de différentes sources, telles que des observations directes des performances passées du vendeur, son expérience avec d'autres entreprises ou les informations communiquées par d'autres parties (le bouche-àoreille), notamment. Il existe plusieurs méthodes pour modéliser la réputation, et ces dernières années, de nombreuses contributions, tant théoriques qu'empiriques, ont été publiées sur ce sujet.

\section{La réputation individuelle}

Si nous nous concentrons sur les informations émanant des transactions passées, il est possible de distinguer trois grandes catégories de modèles pour la réputation individuelle : l'information cachée, l'action cachée et les modèles mixtes (Bar-Isaac et Tadelis, 2008) $)^{5}$.

\footnotetext{
4. Nous étudions ici la réputation des entreprises, mais cette analyse peut s'appliquer à tout vendeur ou agent économique.

5. Cette section se fonde sur Bar-Isaac et Tadelis (2008), qui constitue une bonne introduction à cette littérature. Pour une présentation un peu plus technique et approfondie, voir Mailath et Samuelson (2006) et pour une étude technique récente, voir Mailath et Samuelson (2013).
}

Dans les modèles d' « information cachée pure », le vendeur ne joue pas de rôle actif pour influer sur l'issue de la transaction, mais la qualité de la marchandise dépend cependant des caractéristiques du vendeur (type d'acteur). Dans cette catégorie de modèles, la réputation est modélisée sur la base des croyances du consommateur quant au type d'entreprise auquel il a affaire. Les croyances sont généralement modélisées comme des probabilités (postérieures), et la mise à jour des croyances (suivant le théorème de Bayes) est fonction du succès ou de l'échec de la marchandise, c'est-à-dire de l'histoire personnelle de l'entreprise. La réputation est donc modélisée comme « l'apprentissage pur» du type de vendeur, ce qui est considéré comme une vérité sous-jacente, qui n'est pas manipulable et qui peut être découverte à partir des performances passées.

Dans les modèles d' " action cachée pure », l'entreprise peut être d'un type unique, mais l'incertitude sur la qualité dépend du comportement d'équilibre de l'entreprise. En d'autres termes, la qualité de l'entreprise dépend de ses actions et la réputation est modélisée comme correspondant aux croyances du consommateur à propos du comportement d'équilibre de l'entreprise. Dans cette catégorie de modèle, le fait que les transactions soient répétées permet à la réputation de s'établir et autorise également des sanctions en cas d'écarts. L'approche de la «carotte » et du «bâton » incite les entreprises à trouver le juste équilibre entre les incitations à court terme à transiger sur la qualité et le risque à long terme de perdre des opportunités commerciales.

Dans les modèles « mixtes », qui sont peut-être les plus réalistes, la qualité de la marchandise dépend à la fois du type de vendeur et de ses actions. La réputation est ainsi représentée par les croyances de l'acheteur concernant le type de vendeur et le comportement d'équilibre anticipé pour 
les différents types. Lorsque le vendeur connaît son type, ce qui est le cas dans les modèles de signalement, les types sont en général (au moins) au nombre de deux : un type d'entreprise de nature stratégique, ou opportuniste, qui peut décider de produire de la bonne qualité ou de la qualité inférieure ; et un type enclin à l'engagement, qui choisit toujours le même niveau de qualité.

Les modèles mixtes peuvent ensuite être répartis en deux sous-catégories. Dans les modèles d'imitation, le type enclin à l'engagement produit toujours de la bonne qualité tandis que le type stratégique est le mauvais type qui peut décider de produire de la bonne qualité pour être confondu par les consommateurs avec le bon type. Dans les modèles de séparation, en revanche, c'est le type enclin à l'engagement qui est l'entreprise du mauvais type (que l'on appelle en fait le type «médiocre »), qui produit toujours de la qualité inférieure, tandis que le type stratégique est l'entreprise du bon type, qui peut décider de produire des marchandises de bonne qualité pour se distinguer du vendeur de produits médiocres (de qualité inférieure).

Il convient de noter que, dans tous les modèles, le jeu est répété et donc que "l'on récolte toujours ce que l'on sème ». Cependant, tandis que dans les modèles d'action cachée, les comportements inadéquats s'exposent à des représailles punitives, dans les modèles d'information cachée et dans les modèles mixtes, ce sont les croyances qui sont décisives : après de mauvaises expériences, les consommateurs mettent à jour leurs croyances pour intégrer la plus forte probabilité que la mauvaise qualité persiste et apprennent à reconnaître les entreprises qui vendent de la mauvaise qualité (Bar-Isaac et Tadelis, 2008). L'expérience (ou la qualité) d'aujourd'hui est déterminée par l'action ou par le type de vendeur, mais l'acheteur met à jour ses croyances, et donc l'expérience actuelle influe également sur les transactions à venir.

Dans les modèles de réputation individuelle, les facteurs susceptibles d'expliquer dans quelle mesure la réputation peut être efficace pour préserver un commerce efficient sont peu nombreux. Il y a tout d'abord le degré d'incertitude concernant le vendeur ; ensuite, la vitesse à laquelle les consommateurs apprennent de leurs expériences, par exemple la vitesse à laquelle l'information circule entre les acheteurs. S'y ajoute la patience du vendeur (son taux d'actualisation), puisque plus le vendeur est patient, plus il accorde d'importance à l'avenir. Et enfin, il faut citer les caractéristiques de la demande, qui font que les acheteurs sont plus ou moins sensibles à la réputation.

\section{La réputation collective}

Si le nombre des contributions sur la réputation individuelle est très élevé et ne cesse d'augmenter, celui des études consacrées à la réputation collective est plus restreint (mais s'accrôtt, lui aussi). Tirole (1996) constitue la référence fondatrice, car c'est l'une des premières contributions à modéliser la réputation collective comme la somme des réputations individuelles. Il s'agit d'un modèle à générations imbriquées, avec un jeu d'appariement, où la qualité passée de chaque membre du groupe est observée avec du bruit. Il existe trois types d'agents (honnêtes, malhonnêtes et opportunistes), et la présence du type honnête incite l'opportuniste à se forger une réputation.

Le modèle général est assorti de deux variantes : l'une dans laquelle l'incitation à préserver la réputation vient de la crainte d'une exclusion directe par le partenaire à la transaction, et l'autre dans laquelle cette incitation vient de la crainte d'une exclusion indirecte, par le groupe, par exemple par un licenciement. Ce modèle est plutôt général, mais il est appliqué dans deux 
environnements différents pour expliquer la corruption dans les groupes sociaux et la qualité dans les entreprises (une coopérative de travailleurs).

Il en ressort des résultats différents, mais le plus intéressant est probablement que les réputations individuelles sont déterminées par les réputations collectives, et inversement. Plus l'incitation d'un membre à préserver sa réputation individuelle est forte, meilleure est la réputation du groupe. Les externalités réputationnelles perdurent, et des cercles vicieux peuvent apparaître, conduisant parfois des membres nouveaux d'une organisation à souffrir des choix de leurs aînés bien longtemps après que ces derniers sont partis. S'agissant de la qualité des entreprises, un licenciement de masse (touchant même les travailleurs honnêtes) constitue parfois le seul moyen de remédier à une mauvaise réputation. De plus, l'intensification de la concurrence atténue les incitations individuelles à préserver la réputation et peut donc porter atteinte à la réputation des entreprises.

Winfree et McCluskey (2005) proposent un modèle dans lequel, contrairement à celui de Tirole (1996), le nombre d'entreprises dans le groupe devient important et la qualité individuelle n'est pas observable. La réputation collective repose sur la qualité moyenne passée du groupe, et elle est perçue comme un bien commun. Par ailleurs, comme dans d'autres modèles comportant une action cachée (par exemple, Shapiro, 1983), les consommateurs observent la qualité passée avec un décalage dans le temps ${ }^{6}$. De manière générale, il apparaît (comme dans le cas d'un bien commun) que lorsque le nombre des membres augmente, les incitations pour

6. Les croyances évoluent selon un processus de Markov préspécifié, et non par un apprentissage endogène de la qualité tel qu'une mise à jour bayésienne. chaque individu à adopter un comportement de passager clandestin vis-à-vis de la réputation du groupe s'accroissent. Ces auteurs envisagent deux stratégies possibles pour les membres : avec une norme de qualité minimale, le groupe peut atténuer ou éviter le comportement de passager clandestin, tandis qu'avec des stratégies du déclic, les entreprises individuelles (puisque leur qualité n'est pas traçable) retireraient trop du stock de réputation collective.

Certains articles récents se sont intéressés à la coexistence d'une réputation privée (ou individuelle) et d'une réputation collective. Landon et Smith (1998) estiment l'impact de la qualité des produits et de la réputation sur les prix, et décomposent l'impact de la réputation en effets de la réputation individuelle et collective. Avec un modèle hédonique modifié, ils utilisent des données concernant les vins de Bordeaux et observent que l'impact de la réputation sur les prix est vingt fois supérieur à l'impact de la qualité courante, ce qui laisse à penser que la réputation occupe une place centrale dans la formation des prix. De plus, les indicateurs de la réputation collective jouent un rôle significatif dans la formation des prix, via leur impact sur la qualité attendue, et donc influent sur les prix dans la mesure où ils sont de bons prédicteurs de la qualité. Dans l'ensemble, ces auteurs montrent également que les modèles empiriques qui incluent des variables de substitution pour les réputations individuelles et collectives ont une meilleure valeur prédictive que les modèles tenant compte uniquement de la réputation individuelle ou uniquement de la réputation collective, ce qui justifie d'analyser le double impact de ces réputations.

Costanigro et al. (2010) proposent un modèle hédonique empirique permettant d'analyser conjointement la réputation du produit, celle de l'entreprise et la réputation 
collective ${ }^{7}$. À l'aide de données sur les vins californiens, ils désagrègent la prime de réputation en différentes performances de qualité, en tenant compte non seulement de la qualité moyenne, mais aussi de la constance de la qualité et de la longévité du nom, dans un modèle qui intègre des noms spécifiques et des noms agrégés. Ils montrent que tant la qualité moyenne que la constance de la qualité jouent un rôle important. De plus, la prime de réputation se réduit à mesure que le nom devient plus spécifique, c'est-à-dire lorsque l'on passe de la réputation collective aux réputations individuelles puis à la réputation des produits. Enfin, leurs données montrent que l'utilisation de la réputation collective est importante pour les vins bon marché, tandis que pour les vins plus haut de gamme, le nom spécifique gagne en importance. Ils avancent d'ailleurs que lorsque les consommateurs dépensent davantage pour du vin, le coût d'un mauvais choix, c'est-à-dire de l'achat d'un vin de mauvaise qualité pour un prix élevé, est supérieur, ce qui justifie le coût supérieur que les consommateurs acceptent de supporter pour se renseigner lorsqu'ils passent d'un nom collectif à un nom spécifique.

Costanigro et al. (2012) se penchent plus avant sur la coexistence entre réputation privée et réputation collective et étudient leur double impact sur l'investissement des entreprises dans la qualité. Leur modèle de jeu dynamique intègre Shapiro (1983) et Winfree et McCluskey (2005) comme des cas spéciaux. Ils ont

7. Castriota et Delmastro (2010) étudient les déterminants de la réputation des entreprises en tenant compte de la réputation individuelle et de la réputation collective. En s'appuyant sur des données concernant les vins italiens, ils démontrent l'effet positif de l'âge, de la taille, et des investissements d'une entreprise et de la réputation collective sur la réputation individuelle de l'entreprise. Ils montrent également que, dans les coopératives, plus les membres sont nombreux, moins la réputation est bonne. donc des équations de transition d'état qui donnent une approximation de l'évolution des croyances communes. L'une de ces équations concerne les réputations privées, pour lesquelles l'acheteur met à jour ses croyances sur la base de l'écart entre la qualité réelle et la qualité attendue, avec certains paramètres exogènes pour le décalage temporel de l'information (comme dans Shapiro, 1983) et pour la visibilité de la marque. Dans l'équation concernant la réputation collective, la qualité attendue correspond à la moyenne pondérée de la qualité du groupe, et ce modèle a deux variantes : l'une, comme dans Winfree et McCluskey (2005), dans laquelle tous les membres reçoivent la même pondération ${ }^{8}$, et l'autre dans laquelle certaines entreprises, les « leaders réputationnels », peuvent recevoir une pondération supérieure.

Ces auteurs paramètrent le modèle établi sur la base des données concernant les vins californiens et procèdent à des simulations. Ils confirment que la qualité et les réputations s'améliorent lorsque les consommateurs ont plus de facilité à se renseigner ; cependant, sans coordination, il se peut que l'investissement dans la qualité ne soit pas efficient. À l'aide d'une régression quantile, ils confirment également que les réputations privées deviennent d'autant plus précieuses que les vins sont chers. Enfin, les réputations collectives d'équilibre s'améliorent en présence d'un leader réputationnel (cependant, les différences entre les équilibres coordonnés et les équilibres concurrentiels augmentent avec le poids du leadership).

Menapace et Moschini (2012) étendent le modèle de Shapiro (1983) pour explorer la coexistence de plusieurs réputations collectives sous la forme d'indications

8. Dans tous les modèles mentionnés jusqu'ici, la quantité est la même pour toutes les entreprises et elle est souvent normalisée à l'unité. 
géographiques (IG) axées sur l'utilisation de noms liés à l'origine géographique d'un produit et de marques de commerce privées. Ils établissent un modèle incluant un bien d'expérience, des équilibres concurrentiels et une offre parfaitement élastique. Concernant les indications géographiques (IG), ils comparent deux environnements institutionnels : le dispositif sui generis de l'UE, c'est-à-dire les réputations collectives régies par l'UE (Règlement $\mathrm{CE} / 510 / 2006$ ), qui désigne la région de production et précise certaines règles de production (le cahier des charges, que ces auteurs modélisent comme la norme de qualité minimale), et le système de marques de certification en vigueur aux États-Unis, qui ne désigne que la région de production (par exemple l'American Viticultural Area ou AVA, à savoir la Région viticole américaine).

Ces auteurs observent que les marques de commerce individuelles et les IG collectives constituent des instruments complémentaires pour signaler la qualité. De plus, ils montrent que les IG abaissent le coût de l'établissement des réputations privées. En outre, les consommateurs en bénéficient (en particulier ceux qui apprécient les produits de bonne qualité), car les IG réduisent le coût de la formation d'une réputation pour les entrants également, et donc la valeur des réputations établies. Enfin, si le modèle d'IG sui generis de l'UE et le modèle américain des marques de certification atténuent le problème de l'aléa moral (parce qu'en révélant des informations utiles, ils restreignent les possibilités d'adopter un comportement opportuniste), le modèle choisi par l'UE semble préférable dans la mesure où il révèle davantage d'informations.

Tous ces modèles de réputation collective se rangent dans la catégorie de l'action cachée pure. Celui décrit dans Fishman et al. (2008) constitue une exception notable : c'est un modèle mixte (action cachée et information cachée), dans lequel les entreprises connaissent leur qualité et où les entreprises stratégiques vendent des produits de bonne qualité, c'est-à-dire qu'il s'agit d'un modèle de séparation. Le recours à une marque collective (collective branding) est modélisé comme un groupe de $m>1$ entreprises de grande qualité qui commercialisent ensemble leurs produits sous un nom de marque commun, mais conservent leur autonomie pleine et entière pour les décisions relatives à leur activité et aux bénéfices, et en particulier qui décident à titre individuel si elles investissent ou non dans la qualité.

De manière générale, le retour sur l'investissement dans la qualité dépend du degré d'information des consommateurs à propos des performances passées de l'entreprise : par exemple, avec une communication par bouche-à-oreille, il dépend du nombre de consommateurs qui ont expérimenté le produit dans le passé9. Fishman et al. (2008) partent de l'hypothèse principale que les consommateurs évaluent la qualité de l'entreprise sur la base de leur expérience globale de la marque collective, et pas uniquement d'après leur expérience de ses performances individuelles passées. La marque collective apporte donc davantage d'informations, car un consommateur peut dès lors obtenir, pour chaque membre de la marque, un nombre d'observations égal au nombre de membres de la marque.

Ils montrent ensuite qu'une bonne réputation de marque est plus précieuse pour ses membres qu'une bonne réputation indépendante, c'est-à-dire qu'ils montrent

9. Ainsi, «plus l'entreprise est petite et moins ses clients sont informés de sa qualité passée. Les petites entreprises peuvent donc se trouver incapables d'établir efficacement une réputation individuelle toutes seules, et par conséquent ne seront guère incitées à investir dans la qualité. Ici, le recours à une marque collective peut les sauver » (Fishman et al., 2008, p. 2). 
l'effet de réputation de la marque. Cependant, il existe également un effet d'opportunisme (comportement de passager clandestin) sur l'investissement des autres membres, et donc l'effet global de la marque collective dépend de l'interaction entre ces deux forces opposées. Dans le cas d'un contrôle parfait, tout comportement de passager clandestin est interdit et les tricheurs, c'est-à-dire les entreprises qui n'investissent pas, sont exclus. Dans ce cas simple, l'incitation à investir augmente de manière monotone avec le nombre d'adhérents à la marque et le recours à la marque collective entraîne une amélioration de la qualité. En l'absence de comportement de passager clandestin, lorsque la taille de la marque augmente, les consommateurs ont davantage d'observations sur le type de marque, et par conséquent les incitations à investir sont supérieures à celles des entreprises opérant seules.

Dans le cas d'un contrôle imparfait, qui est certainement plus réaliste, dans lequel la marque n'est pas capable de contrôler les investissements de ses membres individuels et de contraindre ces derniers à investir sous peine d'exclusion, l'effet de réputation doit être supérieur à l'effet d'opportunisme pour que la marque soit incitée à investir davantage dans la qualité que l'entreprise opérant seule. Cela se produit lorsque le groupe est petit et/ou la qualité attendue de l'investissement est élevée. En effet, si la marque est étendue, la marque collective est moins incitée à investir que l'entreprise opérant seule, car la contribution marginale de l'investissement d'un membre individuel à la visibilité et à la réputation de la marque devient négligeable comparé à ce que rapporte un comportement de passager clandestin ${ }^{10}$.

10. Castriota et Delmastro (2010) observent que la réputation de la marque augmente avec le nombre de bouteilles produites mais baisse avec l'augmentation du nombre de producteurs affiliés à la marque.

\section{Des choix critiques pour les marques collectives}

Depuis l'adoption de la réglementation nationale sur les AOC, la législation autorise un groupe de producteurs qui recourent à des techniques de production bien définies et sont situés dans une région donnée à gérer leur réputation collective. Le groupe décide des règles de production, de leur contrôle et des sanctions prévues en cas de non-respect, de critères à remplir pour rejoindre le groupe et des conditions d'exclusion en cas de manquements à ces règles, etc. La marque collective est donc la propriété du groupe, qui la gère selon des principes démocratiques. Les entreprises membres peuvent utiliser la marque collective, seule ou accompagnée d'une marque de distributeur ou d'une marque privée.

Un certain nombre de groupes ont dû faire différents choix qui les ont contraints à prendre des décisions difficiles. De nombreux consommateurs sont conscients de l'existence de ces groupes (par exemple, en tant que consommateurs de certains produits AOP), mais ils ne savent pas grand-chose de leur fonctionnement, sauf dans les rares occasions où les médias en parlent. En effet, il arrive que fuitent dans les médias, peut-être à des fins stratégiques, des informations sur des différends entre membres du groupe ou des choix controversés opérés soit par les groupes qui gèrent l'AOP soit par des membres en désaccord avec les décisions de la majorité (et qui pourraient quitter l'AOP).

\section{Les choix du secteur}

Dans le secteur du fromage, par exemple, la région de production du Camembert est le théâtre de différends au sujet de l'autorisation de l'emploi du lait pasteurisé (au lieu du lait cru) pour la production de l'AOP Camembert de Normandie. Certaines grandes entreprises proposent la pasteurisation, car elle évite les problèmes 
de sécurité des aliments posés par une bactérie particulièrement résistante, la Listeria. En effet, contrairement à bon nombre d'autres germes, la Listeria peut se développer aux basses températures du réfrigérateur, mais elle est détruite par la cuisson et la pasteurisation. Or, pour les partisans du statu quo, dont font partie les petits producteurs de fromage, le lait cru est à l'origine des caractéristiques uniques du Camembert de Normandie. En 2007, à la suite de ce différend, certains producteurs ont quitté l'AOC et produisent désormais $\mathrm{du}$ «Camembert fabriqué en Normandie». Cependant, des poursuites ont été engagées à leur encontre pour « usurpation de notoriété », et ce différend n'a pas encore été tranché par le tribunal (Le Du, 2014) ${ }^{11}$.

Des différends analogues sont apparus lorsqu'un grand distributeur a demandé à des fromagers de fabriquer un Camembert de marque de distributeur. Certains producteurs, comme la Coopérative d'Isigny, y étaient favorables, espérant trouver ainsi de nouveaux débouchés pour leurs produits, tandis que d'autres, soutenus par l'Institut national des appellations d'origine (INAO), craignaient une «banalisation » de la marque Camembert (INA, 1997).

En effet, avec l'évolution du secteur de la distribution et l'importance croissante des marques de distributeur, certaines AOP ont été invitées à produire pour la marque d'un distributeur (ou mises devant le fait accompli lorsque certains membres ont commencé à le faire d'eux-mêmes). La question de savoir s'il fallait « fusionner » la réputation de l'AOP (ou de toute marque collective) avec celle de la marque du distributeur s'est probablement posée pour la première fois avec le « Roquefort Casino ». La réglementation de l'AOC Roquefort n'autorisait pas les distributeurs

11. Le tribunal de Rennes devait rendre sa décision à l'automne 2014, c'est-à-dire après la rédaction du présent article. à produire ce fromage, mais Casino s'approvisionnait en réalité auprès d'un petit fabricant à Roquefort. Comme on le sait depuis longtemps, cette pratique du secteur de la distribution tient au fait que « les indications géographiques ont tendance à attirer ou à rassurer le consommateur » (ANF, 1998).

On rencontre des affaires analogues dans d'autres pays de l'UE et pour d'autres produits agroalimentaires. Ce fut récemment le cas de la Valpolicella, la deuxième plus grande région productrice de vin rouge en Italie. Les entreprises de cette région produisent différents types de vins rouges. Depuis quelques décennies, l'Amarone, un vin puissant qui a beaucoup de corps, est le vin le plus rentable. Son prix dépasse largement celui d'autres vins de la même région et des mêmes cépages.

Historiquement, l'Amarone était produit dans une zone de collines bien délimitée, considérée comme la plus susceptible de donner un raisin d'excellente qualité. Mais, au fil du temps, sous l'effet de l'augmentation de la demande, la zone de production de l'Amarone s'est étendue à d'autres territoires, et en particulier aux vallées en plaine, où les rendements sont supérieurs, mais la qualité peut-être inférieure. Cette situation de facto contraste avec ce qui était prévu, c'est-à-dire avec la règle prévoyant que l'Amarone soit principalement produit dans les zones de collines traditionnelles. Par conséquent, le Consorzio di Tutela, l'organisme qui gère l'AOP Valpolicella, a récemment proposé d'autoriser la production de l'Amarone dans les vallées en plaine (dell'Orefice, 2013b). Les partisans de cette extension avancent que cette règle était due à une coquille dans le cahier des charges original de 1968. En outre, l'abolition de cette règle reviendrait à reconnaître la réalité de la production, car un grand volume d'Amarone provient déjà de ces zones (dell'Orefice, 2013a). 
Cependant, de nombreuses entreprises, en particulier celles qui ont massivement investi dans la technologie de production de l'Amarone et qui sont plus connues dans le monde, sont opposées à cette modification et affirment avec véhémence que la production de l'Amarone doit rester confinée aux zones de collines, pour des raisons de qualité (Guerrini, 2013a) ${ }^{12}$. Certaines ont également menacé de quitter l'AOP (Guerrini, 2013b). Le 10 mai 2013, à la suite d'une décision prise à l'unanimité par son conseil d'administration, l'assemblée des producteurs a confirmé à la majorité l'abrogation de la règle ${ }^{13}$.

\section{Les choix de modélisation}

Ces exemples sont loin d'être les seuls, mais ils constituent les cas les plus connus de choix critiques que doivent parfois opérer des marques régionales. La plupart de ces situations de différend au sein des marques présentent certains points communs : un groupe d'entreprises utilise une marque régionale (l'AOP) et ce groupe se compose d'entreprises hétérogènes. En simplifiant un peu, on peut dire qu'il se compose d'entreprises de qualité supérieure et d'entreprises de qualité inférieure. L'AOP peut leur permettre de saisir conjointement des opportunités commerciales, comme un nouveau marché ou

12. Sandro Boscaini, propriétaire de Masi Agricola, a déclaré : « Je ne suis pas certain que le Valpolicella doive être produit en dehors des collines. La zone Classico est historiquement une zone de petits producteurs, et le reste est composé à $90 \%$ de coopératives, qui essaient d'atténuer le plus possible les différences entre la région d'origine et la nouvelle zone. Je ne dis pas qu'ils ne sont pas capables de produire du bon vin. Mais [l'extension de la région $\mathrm{AOC}]$ a été une catastrophe pour le Valpolicella, qui a perdu en qualité » (Rand, 2013).

13. Cette décision n'a probablement pas été une surprise, étant donné que « le système de vote, qui repose sur le quota de production de raisin, donne un avantage aux plus gros producteurs qui étaient favorables à l'abrogation » (Costanzo, 2013). l'adoption d'une innovation susceptible de conduire à une technologie qui réduise les coûts ou améliore la qualité (qualité opposée à tradition?), etc.

Or, pour saisir cette opportunité, le groupe doit prendre une décision, et donc modifier son cahier des charges. Certaines entreprises sont favorables à l'adoption et à la mise en œuvre des changements nécessaires pour saisir cette opportunité, alors que d'autres y sont opposées, car elles redoutent probablement que cette décision ait des effets délétères sur la réputation collective. Les décisions relatives aux règles (voire aux sanctions en cas de non-respect des règles) peuvent être prises sur une base démocratique, c'est-à-dire par un vote à la majorité, par lequel le groupe décide de saisir (ou non) l'opportunité commerciale. Dans ce nouvel équilibre, les entreprises du groupe peuvent accéder à de nouveaux marchés, adopter des innovations, etc. Toutefois, si certaines seront gagnantes, d'autres seront peut-être perdantes et pourront même décider de quitter l'AOP.

\section{Hétérogénéité}

Les modèles examinés ne présentent pas l'intégralité des caractéristiques essentielles de ces cas. Premièrement, les membres des groupes sont hétérogènes. Certains sont de petits producteurs, d'autres de gros producteurs, comme dans le secteur du vin. Certains sont intégrés verticalement, c'està-dire qu'ils produisent leur propre vin, tandis que d'autres livrent leurs produits à une usine de transformation privée ou à une coopérative. Ils fabriquent des produits de plus ou moins bonne qualité selon leur emplacement (collines ou plaines dans certaines régions), les technologies adoptées, leurs traditions ou leur savoir-faire. L'hétérogénéité des membres explique qu'ils peuvent y trouver des incitations et des bénéfices différents, d'où leurs points de vue et leurs décisions.

À notre avis, les modèles réalistes doivent tenir compte de cette hétérogénéité 
afin que les prévisions correspondent davantage aux données empiriques. Par exemple, on pourrait s'attendre à ce que les groupes plus homogènes soient moins enclins aux différends. Ainsi, certains « mauvais » équilibres ou résultats ne s'expliquent pas nécessairement par la taille du groupe, comme ont pu le suggérer Fishman et al. (2008), mais par le poids relatif des bons et des mauvais acteurs au sein du groupe. En effet, nonobstant sa taille restreinte, un petit groupe peut rencontrer des problèmes du fait de son hétérogénéité et, inversement, un groupe comptant beaucoup de membres peut fonctionner relativement bien, car il est homogène.

En outre, un examen plus poussé de l'interaction entre la réputation privée, la réputation collective et l'hétérogénéité peut expliquer les résultats différents observés dans le secteur. Lorsque la majorité est constituée d'entreprises trop conservatrices ou fabriquant des produits de qualité inférieure, des entreprises privilégiant la qualité peuvent préférer ne s'appuyer que sur leur propre réputation (car leur palmarès individuel est bien supérieur à celui du groupe) et donc quitter le groupe (et cesser d'utiliser la marque régionale). En Toscane, par exemple, dans les années 1970, certains producteurs ont quitté l'AOP Chianti (qui imposait le coupage de cépages Sangiovese avec des cépages locaux) afin de tester un coupage avec d'autres cépages internationaux, comme le Cabernet ou le Merlot. Ces expérimentations ont conduit à la production des vins appelés « Super Toscans », qui ne pouvaient à l'origine être produits qu'en dehors de l'AOP, en tant que vins de table. Finalement, quelques producteurs ont lancé une toute nouvelle AOP (comme Bolgheri), en produisant des vins qui sont devenus plus réputés (et plus chers) que les vins AOP initiaux.

\section{Prise de décision interne}

Si certaines décisions relatives aux marques régionales et collectives sont prises par les autorités de l'UE ou des pays, beaucoup d'autres relèvent du groupe de producteurs qui gère la marque. En règle générale, ces groupes obéissent à des processus décisionnels démocratiques. Cependant, les règlements ou dispositions statutaires (par exemple, faut-il une majorité simple ou qualifiée pour modifier certaines règles ? Les droits de vote sont-ils proportionnels à la production ou reposent-ils sur le principe « une entreprise, une voix »?), les structures de gouvernance (au sein d'un grand groupe, les membres votentils directement ou par le biais de comités élus ?), la composition du groupe (la majorité est-elle composée de petits ou de gros producteurs, de producteurs de qualité supérieure ou inférieure ?) sont autant d'éléments susceptibles d'influencer les résultats et les équilibres du secteur. Par exemple, les équilibres atteints différeront suivant que les petits producteurs (ou les producteurs de qualité inférieure, ou les producteurs traditionnels, etc.) ont ou non la majorité, et en fonction de la liberté dont ils disposent pour décider des règles en fonction de leur intérêt. En général, toutes choses étant égales par ailleurs, les décisions prises avec des règles ou des structures de vote différentes peuvent produire des résultats très différents. C'est pourquoi il importe de tenir également compte de ces aspects.

\section{Tradition contre innovation}

Le cas des vins « Super Toscans » est également intéressant parce qu'il illustre le dilemme entre « tradition et innovation ». Ces expérimentations avaient pour objectif de produire des vins de garde, comme les Bordeaux, mieux connus. Certains producteurs ont essayé avec le Cabernet et le Merlot uniquement. C'est le cas du Sassicaia, qui est produit sur un terroir similaire au vignoble des Graves, une subdivision du vignoble de Bordeaux. Dans le cas du Tignanello, les producteurs ont utilisé le principal cépage local, 
le Sangiovese, seul ou associé à $15 \%$ de Cabernet Sauvignon.

Certaines de ces expérimentations ont été jugées intéressantes et compatibles avec le cahier des charges. Elles ont par la suite été intégrées dans les règles du Chianti. D'autres producteurs ont, quant à eux, préféré continuer à vendre ces vins comme vins de table. Toutefois, ces innovations ont permis de montrer que le Sangiovese présentait un immense potentiel pour la production de vins de garde, confirmant, un siècle plus tard, l'intuition et les choix du Baron Ricasoli.

\section{Une production limitée}

Lorsque l'on analyse la réputation collective, il peut s'avérer utile de tenir compte du choix des niveaux de production, en termes de quantité produite ou de surface cultivée. Lorsqu'elles cherchent à déterminer le niveau de qualité optimal, la plupart des contributions s'intéressent aux entreprises qui produisent un produit unique ${ }^{14}$. Or on sait bien que différentes pratiques peuvent augmenter les rendements mais nuire à la qualité. Dans la production de vin, par exemple, « si un viticulteur choisit des clones à rendement élevé, ne les taille pas correctement ou emploie trop d'engrais, la

14. Fleckinger (2007) fait exception. Il étudie un oligopole de Cournot avec des «biens d'expérience » différenciés de façon endogène, dans lequel les entreprises se font concurrence tant sur la qualité que sur la quantité. Cette contribution analyse le cas où les consommateurs connaissent la qualité moyenne offerte par un ensemble de producteurs, mais pas celle d'un producteur donné. Il s'agit d'une situation intermédiaire entre les cas extrêmes de l'information parfaite et de l'asymétrie de l'information. Elle montre que la concurrence entraîne une augmentation de la quantité commercialisée totale, mais une baisse de la qualité, ce qui peut être dangereux pour les consommateurs (lorsque les normes de qualité obligatoires sont peu élevées). Par conséquent, les normes de qualité et la concurrence sont complémentaires au sein d'une marque collective. En outre, la réglementation de la quantité est la meilleure des mesures comportant un seul instrument. qualité en pâtira inévitablement » (Johnson et Robinson, 2001) ${ }^{15}$. Ainsi, les fromagers de Basse-Normandie se sont récemment demandé s'ils devaient «produire plus ou produire mieux » (Le Du, 2014).

De nombreuses marques régionales, si ce n'est toutes, délimitent également la zone de production et les producteurs ne sont autorisés à apposer la marque régionale qu'à la condition qu'ils opèrent dans cette zone, sur la foi de l'affirmation ou de la supposition que l'appartenance au territoire délimité est le gage d'une qualité meilleure ou reconnaissable. À notre connaissance, la seule contribution qui ne s'intéresse pas à un produit unique est celle de Menapace et Moschini (2012). Dans leur modèle, l'offre est parfaitement élastique. Cependant, ils reconnaissent que leurs résultats, en particulier le fait que les consommateurs bénéficient d'effets positifs tandis que les producteurs n'ont que des effets négligeables, voire négatifs, pourraient être en partie différents dans un modèle intégrant une zone de production bien délimitée.

En effet, une hausse des prix induit une augmentation des rentes pour les facteurs fixes, par exemple avec le renchérissement des terres. Les écarts de prix des biens fonciers entre les exploitations agricoles à l'intérieur des zones AOP et celles à l'extérieur peuvent être considérables, et souvent sources de différends. Ainsi, en 2007, les terres se trouvant dans la zone du Champagne pouvaient valoir jusqu'à 50 fois plus que les terres ordinaires de la région alentour. La délimitation des zones de production a donné lieu à des différends (qui ont pu aller jusqu'au procès).

15. C'est probablement pour ces raisons que de nombreux cahiers des charges pour les vins et les fromages AOP limitent le rendement par hectare ou par vache, ou qu'il existe d'autres règles, comme l'interdiction du maïs-ensilage, qui limitent les rendements, vraisemblablement dans un souci d'amélioration de la qualité. 
Les producteurs situés dans des villages à la périphérie immédiate de la zone officielle du Champagne ont fait valoir qu'ils proposaient une qualité comparable (sinon meilleure) à celle offerte par les producteurs implantés dans la région officielle du Champagne (Jefford, 2008) ${ }^{16}$. Nous pensons qu'il serait intéressant d'étudier les effets des différents niveaux de production (ou des délimitations des zones de production) sur la réputation et, de là, sur la qualité.

\section{Coûts de l'établissement de la réputation}

Les coûts de l'établissement de la réputation ont une incidence différente sur des entreprises selon leur taille. Il a été démontré que la réputation privée bénéficie davantage aux entreprises de grande taille (Rob et Fishman, 2005). Ces dernières peuvent en effet trouver un avantage à utiliser des marques privées et ainsi recourir à deux stratégies (au moins) : leur propre marque et la marque collective. Le problème est différent pour les petites entreprises, pour lesquelles le coût de création d'une marque privée bénéficiant d'une réputation individuelle peut se révéler prohibitif. Elles peuvent alors trouver intéressant de partager les coûts d'une marque à plusieurs.

En effet, la réglementation de l'AOP s'est révélée utile en permettant à des producteurs, même petits, de profiter d'une réputation bien établie (Bureau et Valceschini, 2003). De récentes données empiriques confirment que les petites entreprises qui adoptent volontairement une AOP dans la filière des produits laitiers ont une espérance de vie plus longue que les grosses entreprises, qui peuvent bénéficier davantage d'économies d'échelle ou de leur propre marque de qualité (Bontemps

16. Pour certains commentateurs, l'extension de la zone de Champagne à d'autres villages pourrait même entraîner une amélioration de la qualité de la production (voir, par exemple, Jefford, 2008). et al., 2013). Même si l'AOP exerce un impact moins important que la taille (c'està-dire que les grandes entreprises ont une probabilité plus élevée de subsister dans le secteur), l'avantage que présente l'AOP pour les petites entreprises est significatif. La réputation collective peut ainsi améliorer la compétitivité des petites entreprises agroalimentaires. Reste toutefois à voir la question de la répartition des coûts (voir, par exemple, Crespi et Marette, 2001).

\section{Création de valeur grâce \\ à l'intégration verticale}

Lorsque la réputation collective est bonne, elle peut attirer des consommateurs et servir à préserver la valeur dans le cadre d'une intégration verticale. Un exemple récent, extérieur au secteur agricole, illustre le rôle et les effets que la réputation collective peut avoir à cet égard. Une émission télévisée diffusée récemment (Dielhenn, 2014) a montré les choix différents qui ont été opérés dans la coutellerie artisanale française.

À Thiers, la fabrication de lames est une très ancienne tradition. D'après des recherches archéologiques, cette activité était florissante dès le XIII ${ }^{\mathrm{e}}$ siècle. Depuis quelques décennies, fortes de cette longue tradition et de la diversification de la production, les nombreuses coutelleries présentes à Thiers produisent la majorité des lames et des couteaux français (Barriquand, 2008). En particulier, les coutelleries de Thiers sont depuis longtemps les principaux fournisseurs de Laguiole. En 1994, les entreprises locales ont fait enregistrer Le Thiers, marque collective gérée par la Confrérie du Couté de Tié, groupe d'entreprises qui accordent le droit d'utiliser la marque aux coutelleries respectant le cahier des charges. Lorsqu'une entreprise obtient ce droit, elle peut apposer le logo de la marque collective sur la lame à côté de son propre nom.

Laguiole n'a pas des origines aussi anciennes (cette entreprise existerait depuis 
deux siècles, mais la production a été véritablement relancée en 1987), mais elle est plus connue en France et à l'étranger. «L'abeille de Laguiole » est une marque régionale plus connue (bien qu'informelle). Depuis quelques décennies, de nombreuses entreprises ont toutefois opté pour le bas de gamme, et externalisent leur production en Asie, tandis que quelques rares autres, comme la Forge de Laguiole, ont investi dans la qualité. Ces dernières ont créé des marques individuelles qui cherchent à tirer parti de la réputation commune et à accroître leur valeur par une intégration verticale de toutes les phases de la production, y compris celle des lames, qui était traditionnellement externalisée (pour $70 \%$ environ à des forges de Thiers) (Le Parisien, 2013).

Pour renforcer la création de valeur dans ces deux régions, les entreprises fabriquant des produits de qualité ont donc opté pour l'intégration verticale. Ainsi, à Thiers, en aval, les entreprises se sont mises à fabriquer les autres parties du couteau, afin de vendre non plus seulement des lames, mais des couteaux entiers. Et, à Laguiole, en amont, elles se sont mises à forger leurs propres lames. Cependant, si Thiers a choisi une approche coordonnée pour bâtir une réputation collective à partir d'un savoir-faire vieux de plusieurs siècles, Laguiole suit, de son côté, une trajectoire non coordonnée, ponctuée de différentes initiatives, dans l'optique d'utiliser la réputation collective la plus connue.

Le différend qui oppose Thiers à Laguiole présente un autre aspect intéressant : les entreprises de Laguiole ont récemment essayé d'obtenir ensemble l'Indication géographique protégée pour leurs couteaux. Or les autorités françaises ont jusqu'ici reconnu la contribution de Thiers en incluant les deux zones dans la définition de la zone de production relevant de l'IGP. Ce différend a donné lieu à des manifestations et à des actions en justice
(La Dépêche, 2014). Les suites données à ce différend ainsi que la comparaison des stratégies de gestion de la marque collective déployées par Thiers et par Laguiole pourront aider à comprendre comment les chaînes verticales permettent de mieux préserver la valeur.

\section{Quelle (auto ?) régulation ?}

Pour mieux comprendre les différends et les équilibres observés, il peut être nécessaire de prendre en compte certaines des caractéristiques manquantes évoquées plus haut, mais également de participer, aux côtés des pouvoirs publics, à l'élaboration de la réglementation. Il se peut que les autorités s'abstiennent d'intervenir dans certains domaines afin de ne pas limiter la liberté d'entreprise. En revanche, elles pourraient se pencher sur d'autres aspects pour en tirer des indications utiles et intéressantes. Il existe au moins trois grands ensembles de questions qui méritent d'être explorées.

\section{Les questions}

\section{Les contrôles}

Le premier ensemble de questions a trait au choix du système optimal pour contrôler la production et la qualité des groupes. Il existe plusieurs solutions. Les unes consistent à recourir à des tierces parties privées, comme dans les pays anglo-saxons ou pour tel ou tel label ou telle ou telle certification de conformité. D'autres font appel à plusieurs organismes publics. En France par exemple, l'INAO, le ministère de l'Agriculture, les services vétérinaires et la Direction générale de la concurrence, de la consommation et de la répression des fraudes contrôlent les AOP (Assemblée nationale, 1998). D'autres encore reposent sur un seul organisme public, qui représente le plus souvent les producteurs ou les consommateurs ou les agences de santé publique. Enfin, dans certains cas, ce sont les entreprises de l'AOP elles-mêmes qui 
peuvent être habilitées à vérifier que toutes celles qui utilisent une marque respectent bien les règles ${ }^{17}$.

Il convient d'examiner ce qui se passe quand les entreprises qui gèrent l' $\mathrm{AOP}$ sont chargées des contrôles ou des sanctions ${ }^{18}$. Doit-on s'attendre à un équilibre différent si les contrôles ne relèvent pas d'un organisme public, et les contrôles et sanctions différeront-ils suivant le type d'entreprise majoritaire au sein du groupe ? On pourrait avancer que, lorsqu'un groupe est constitué essentiellement par des entreprises proposant des produits de qualité médiocre, en raison de facteurs historiques ou de mauvais choix opérés dans le passé, il risque d'en résulter pour l'AOP un mauvais équilibre qui persistera et, entre autres, moins d'investissements dans la qualité. De plus, il se peut que les règles soient plus laxistes, ou les contrôles/sanctions moins sévères, parce qu'ils ont davantage de chances d'être politiquement plus acceptables sur le long terme. Certaines entreprises proposant de la qualité peuvent préférer quitter le groupe ou promouvoir presque exclusivement leur marque privée. En revanche, quand un groupe est créé essentiellement par des entreprises produisant de la qualité, on constate généralement que sa réputation est meilleure (et qu'il investit davantage), mais aussi qu'il applique plus strictement

17. C'est par exemple le cas en Italie avec le D. $L g s$ (décret législatif) $n^{\circ} 61 / 2010$.

18. Sur la base d'un modèle incluant des agents homogènes, l'aléa moral et des contrôles publics imparfaits, Saak (2012) examine deux autres mécanismes qui visent à discipliner les membres d'une marque collective. Le premier repose sur des normes sociales (sanctions par les pairs), qui répriment les manquements individuels mis au jour via les contrôles locaux effectués par les pairs. Le second mécanisme concerne les peurs alimentaires, c'est-à-dire la méfiance de la population à la suite d'incidents isolés (mauvaise qualité de certains aliments), qui ont toutefois un effet positif dans la mesure où elles font également peur aux producteurs et incitent au respect des normes dans l'ensemble du secteur. les règles. De leur côté, les entreprises qui proposent des produits de faible qualité peuvent considérer que ces règles sont relativement coûteuses, mais décider de ne pas sortir de l'AOP, car elles y bénéficient de la bonne réputation collective ${ }^{19}$.

\section{La «forme optimale » \\ de la réputation collective}

Un deuxième ensemble de questions porte sur la structure optimale du système des différentes appellations et sur la relation entre réputation privée et réputation collective. Dans le secteur du vin par exemple, tous les systèmes d'appellation en place dans l'UE reposent sur le principe de la pyramide : on trouve à la base le « vin de table » et le « vin de pays » (les vins de table de l'UE), puis le « vin délimité de qualité supérieure », et enfin, au sommet, l'appellation d'origine contrôlée, ou AOC (les vins de qualité produits dans des régions de l'UE bien délimitées). Cependant, même à l'intérieur de ce système commun, il existe différents modèles, tant en France que dans d'autres pays.

Prenons le cas de l'AOP dans les grandes régions vitivinicoles françaises. En Bourgogne, il s'agit d'un système hiérarchique, qui correspond en fait à une classification qualitative des quelque 1000 parcelles (les climats) disponibles. Les 33 meilleures parcelles sont les Grands Crus (2 à $3 \%$ environ de la production), dont les producteurs ont le droit de citer uniquement le nom du climat, par exemple Romanée ${ }^{20}$. Les climats qui arrivent en deuxième position, au nombre d'environ 500, sont les Premiers Crus, qui

19. Cela peut expliquer aussi pourquoi il pourrait être préférable de confier les contrôles ou les sanctions à des tierces parties, comme le prévoit, par exemple, 1'UE dans les Règlements n ${ }^{\circ}$ 882/2004/ CE, 1234/2007/CE, 607/2009/CE et 1308/2013/ UE.

20. L'utilisation du seul nom du Grand Cru, sans autre nom collectif, est considérée comme un signe de qualité distinctif. 
représentent moins de $20 \%$ de la production et pour lesquels le nom du Premier Cru ainsi que celui du village où ils sont situés peuvent être utilisés. Viennent ensuite les appellations Village (on en dénombre plus de 50, à peu près $30 \%$ de la production $^{21}$ ). Enfin, les appellations régionales ou de district, «Bourgogne » par exemple, au nombre de 22 , représentent environ la moitié de la production de qualité inférieure. Leurs producteurs peuvent uniquement mentionner le nom de la région ou du district sur l'étiquette de la bouteille. Ces climats sont généralement situés à l'ouest de la route nationale 74 .

Dans le Bordelais, la célèbre classification en crus (du premier au cinquième cru), repose sur la hiérarchie de 1855 , qui classait des «Châteaux » (domaines viticoles) et non des parcelles. Cette hiérarchie incluait essentiellement des domaines du Médoc (exception faite du Château Haute-Brion, un domaine du vignoble des Graves) et elle est restée quasiment inchangée au fil des ans, avec peu de nouveaux entrants et quelques sortants. De plus, peu d'autres classifications concurrentes sont apparues entre-temps (Saint-Émilion, Cru Bourgeois, etc.). Certaines sont également actualisées tous les dix ans environ, mais il n'y a pas de référentiel commun.

Pour les vins de Bourgogne comme pour ceux de Bordeaux, la classification a été établie en 1855, à partir d'informations émanant principalement des négociants, lesquels communiquaient des prix qui reflétaient probablement la qualité des vins. Cependant, en Bourgogne, les exploitations agricoles étaient très fragmentées, et la qualité du Pinot Noir extrêmement

21. Les producteurs peuvent utiliser le nom du village et, éventuellement, le nom du climat, mais en lettres plus petites. Au fil du temps, les producteurs des appellations Village se sont mis à ajouter le nom du cru le plus connu, et, ainsi, à employer un double nom, c'est-à-dire les deux noms séparés par un tiret. variable d'une parcelle à l'autre. En outre, l'analyse des déterminants de la qualité et la cartographie de la zone étaient pratiquées depuis longtemps. Les négociants achetaient des vins provenant de différentes régions de la Bourgogne et ils ont appris à différencier la qualité en fonction du climat. Les prix reflétaient donc le potentiel qualitatif de différentes parcelles. Pour résumer, on peut considérer qu' « aujourd'hui encore, la validité globale de la hiérarchie reste soutenue par le marché » (Lewin, 2010).

Dans le Bordelais, les vins provenaient de différents domaines, qui n'étaient pas associés à des parcelles spécifiques, et la classification de 1855 constituait un « arrêt sur image » des prix atteints par les Châteaux les années précédentes. Néanmoins, ces vins n'ont plus grandchose à voir avec le Claret commercialisé dans la première moitié du $\mathrm{XIX}^{\mathrm{e}}$ siècle. Qui plus est, nombre de Châteaux ne sont plus produits sur les mêmes terres qu'en 1855. C'est pourquoi certains observateurs estiment que le système de classification des vins de Bordeaux est davantage un outil de marketing, voire qu'il « ne correspond pas du tout à la réalité, ce qui ne peut que nuire à la réputation des Bordeaux » (Lewin, 2009).

À notre avis, le système de classification des vins de Bourgogne et sa capacité à prendre en compte et à inclure les différentes appellations, et donc la réputation, est tout à fait intéressant. Le principe de la pyramide, avec, à la base, les régions et les vins de qualité inférieure, est également appliqué aux AOP, d'où une subdivision plus fine, en différents niveaux de qualité : les AOP régionales à la base et les Grands Crus au sommet. Les Grands Crus peuvent bénéficier de la réputation géographique commune (probablement parce que les consommateurs connaissent bien la typologie des vins) et de leur réputation individuelle (seuls les meilleurs peuvent 
faire usage de leur seule appellation), signe d'une qualité supérieure. Et les moins performants peuvent bénéficier des retombées positives des meilleurs, qui ont fait la notoriété du vin concerné. Ce système semble donc un bon moyen de faire concorder les incitations, et un bon compromis pour éviter les conflits entre réputation individuelle et réputation collective.

Certains différends récents dans le secteur du vin en Italie pourraient apporter des arguments laissant à penser que le système de classification des vins de Bourgogne est préférable à un système plus « horizontal », c'est-à-dire relativement étendu et homogène. Un bon exemple est celui du Chianti Classico AOP, avec l'introduction récente, non sans controverses, d'une catégorie supérieure, la « Grande selezione », qui, dans cette appellation, a été spécifiquement créée pour les vins de propriété22. Il semblerait donc que des catégories supérieures supplémentaires soient nécessaires pour prendre en compte l'hétérogénéité des vins à l'intérieur de cette appellation. En effet, dans le passé, très peu de producteurs de Chianti avaient choisi de faire figurer leurs meilleurs vins dans la catégorie vin de pays, une catégorie inférieure (Krebiehl, 2013).

\section{La réputation individuelle Grand Cru ou Domaine?}

En principe, une structure plus nettement « verticale » serait préférable lorsqu'il existe de grandes différences de qualité (hétérogénéité). Mais comment déterminer le degré optimal de « verticalisation » et y parvenir ? Le cas des vins de Bourgogne, et d'autres vins AOP qui ont appliqué ce principe, tels que le Barolo AOP, dans le

22. Il convient de noter que le Chianti AOP est déjà subdivisé en deux zones : celle du Chianti Classico, qui se compose des premiers villages englobés dans la région de production officielle, et celle du Chianti, qui regroupe les villages inclus ultérieurement.
Piémont, et ses menzioni geografiche aggiuntive (mentions géographiques additionnelles), à savoir la possibilité d'ajouter le nom du village et de la sous-région à l'appellation Barolo (Rinaldi, 2012), montre que différentes catégories peuvent gagner à être regroupées dans un système (régional) collectif qui permet le partage de la réputation au niveau de la région, du district, du village, voire des parcelles.

Dans d'autres cas, par exemple pour le Valpolicella, on constate que la mise à profit conjointe de la réputation collective et de la réputation individuelle permet de prendre en compte les différences de qualité au sein d'une même AOP : la réputation collective, plus large, peut désigner des aspects généraux du produit, tels que sa typologie, tandis que la marque privée signale au marché que le producteur fait partie d'une catégorie supérieure.

La principale différence tient au fait que l'établissement d'une réputation privée peut nécessiter des investissements plus coûteux que la participation à une réputation collective dans la catégorie supérieure. Les grandes entreprises seront donc mieux à même de se forger une réputation privée. Par conséquent, la même forme ou structure de réputation collective peut favoriser la multiplication des marques privées dans un secteur comptant de grandes entreprises. Ce constat semble rejoindre celui, par exemple, de Costanigro et al. (2010 et 2012) pour le vin californien.

\section{Les choix de modélisation}

Dans un modèle stylisé, il peut être intéressant d'examiner les effets des choix possibles sur la qualité réelle et perçue (et, donc, sur la réputation), au niveau de chaque entreprise et au niveau collectif. Les articles que nous avons brièvement passés en revue constituent un bon point de départ pour étudier ces différents cas, et tout particulièrement pour modéliser les interactions entre réputation privée 
et réputation collective. Dans Fishman et al. (2008), l'effet de la réputation collective repose principalement sur le passé du groupe, et peu sur celui des différentes entreprises membres. En d'autres termes, dès lors qu'une entreprise fait partie d'une marque collective, sa réputation dépend presque exclusivement des performances de toutes les entreprises du groupe. D'autres études, notamment Costanigro et al. (2012), prennent en compte à la fois le passé du groupe et le passé individuel, mais doivent recourir à des simulations pour obtenir des résultats.

Si l'on veut modéliser les interactions entre les différents niveaux de réputation, il pourrait être utile de combiner la réputation du groupe et la réputation individuelle (performances passées). Une question se pose alors : est-il possible de modéliser le choix qui s'offre à une entreprise, à savoir soit « sortir » de l'AOP, c'est-à-dire établir une réputation privée, soit « progresser » au sein de l'AOP ? Une subdivision plus fine de l'AOP, par exemple avec un équivalent de la mention Grand Cru, pourrait inciter les entreprises proposant de la qualité à rester dans l'AOP, surtout les petites qui, sinon, devraient supporter les coûts liés à l'établissement d'une réputation privée.

Pour finir, Fishman et al. (2008) modélisent la réputation collective en utilisant un modèle de séparation : le groupe composé des entreprises qui proposent de la qualité établit la marque collective dans l'objectif de se différencier des entreprises produisant une qualité inférieure, c'est-àdire extérieures à l'AOP. Il semble toutefois qu'il existe aussi des cas d'imitation au sein même d'une marque, lorsque des entreprises de la marque qui produisent une qualité inférieure, par exemple celles qui recourent à une appellation Village, tentent de profiter de la réputation d'un climat de qualité supérieure (tel qu'un Premier Cru) en accolant le nom de ce cru à celui du village. C'est apparemment ce qui s'est passé en Bourgogne ${ }^{23}$. En étendant l'approche de Fishman et al. (2008), ou d'autres approches, pour modéliser les différentes incitations à se différencier des produits de qualité inférieure et à imiter les produits de qualité supérieure, on pourrait obtenir des informations utiles sur ces problèmes et sur des problèmes connexes. En outre, des travaux plus théoriques seront nécessaires si l'on veut modéliser les interactions entre réputation collective et réputation individuelle.

\section{$*$ \\ * *}

On peut coordonner les activités économiques par différents moyens. L'un d'eux, assez fréquent sur les marchés agricoles, est la réputation collective. En effet, étant donné la demande de produits différenciés et de qualité, le rôle du secteur de la distribution et la reconnaissance des marques régionales par les pouvoirs publics, il est fréquent que les producteurs partagent une marque, tout en restant des entreprises par ailleurs autonomes.

Ces efforts collectifs permettent aux producteurs agricoles d'être plus aisément reconnaissables collectivement et, souvent, d'atteindre plus facilement les consommateurs, mais aussi, parfois, d'obtenir de meilleures conditions de commercialisation auprès des chaînes de distribution.

23. Par exemple, à Barolo, en Italie, à la suite d'une action en justice, la société Marchesi di Barolo a récemment été autorisée à ajouter le nom du terroir (Cannubi) sur l'étiquette des bouteilles de ce vin. Or elle n'est pas à proprement parler située dans la zone de production «Cannubi Cannubi », le Cru le plus connu (Rinaldi, 2012). Certains observateurs craignent qu'à terme, les consommateurs fassent les frais de la dernière décision de justice en date, qui a accordé à cette entreprise le droit d'imiter (d'être confondue avec) un Cru plus prestigieux. En effet, la plupart des consommateurs ignorent que le Marchesi di Barolo Cannubi provient d'une parcelle spécifique, exposée au Nord (Suckling, 2013), et non de la parcelle sur laquelle est produit le Cru le plus connu (Rinaldi, 2013). 
En répartissant les coûts entre un grand nombre d'entreprises, une marque collective constitue un moyen d'accéder à des marchés différenciés. De plus, les petites entreprises, qui, sinon, peineraient à acquérir une réputation individuelle, peuvent ainsi trouver « démocratiquement » une voie d'accès à des marchés de niche. De fait, quand une marque collective est bien gérée, elle améliore la compétitivité et les perspectives de survie pour nombre de petites entreprises produisant de la qualité.

Cependant, les marques collectives sont des organisations complexes, qui peuvent améliorer la compétitivité mais qui vont également être confrontées à des choix difficiles. Des opportunités nouvelles apparaissent et apparaîtront, telles que de nouveaux marchés, de nouvelles technologies, une évolution de la demande des consommateurs ou de nouveaux défis dans un secteur de la distribution en mutation perpétuelle et toujours plus exigeant. À court terme, il pourrait être crucial de saisir ces opportunités, surtout lorsque les prix sont bas sur le marché. Néanmoins, à long terme, l'entrée sur de nouveaux marchés ou l'identification de nouveaux débouchés pourrait mettre à mal, de façon plus insidieuse, la réputation.

Nous avons examiné quelques-uns des problèmes que les marques collectives rencontrent lorsqu'elles cherchent à saisir des opportunités de marché. Avec certaines marques collectives, la qualité est souvent associée à des pratiques traditionnelles, et, par conséquent, l'innovation est perçue comme une menace pour ces traditions. Cependant, étant donné que l'innovation peut aussi apporter de meilleures solutions pour réinterpréter et valoriser ces traditions, la bannir serait délétère pour tous les acteurs. S'il est possible de choisir, mieux vaut probablement partager une réputation collective avec des entreprises similaires, par exemple grâce à un zonage du terroir sur la base de critères scientifiques. Ce zonage étant toutefois susceptible de restreindre la région de production, il risque d'accroître non seulement les incitations à investir dans la qualité, mais aussi les rentes des entreprises incluses, au détriment des autres et des consommateurs, avec pour conséquence une multiplication des différends entre la marque et les parties prenantes extérieures.

Si le groupe de producteurs membres d'une marque collective est hétérogène, des litiges peuvent survenir en son sein, comme le montrent périodiquement les médias. Certaines configurations de marques semblent plus appropriées que d'autres pour faire concorder les incitations entre des membres hétérogènes, mais il peut s'avérer nécessaire de le vérifier au cas par cas. Faut-il tenir compte de l'hétérogénéité avec une marque collective présentant une subdivision plus verticale, comme pour les vins de Bourgogne, ou via l'interaction entre réputation collective et réputation privée ? Cela peut dépendre de la structure du secteur. Dans le cas de petites entreprises, une marque collective plus différenciée peut constituer un bon moyen de faire concorder les incitations pour toutes les entreprises ; d'un autre côté, dans un secteur où opèrent des entreprises plus grandes, des marques privées peuvent venir naturellement compléter les marques collectives.

En outre, la distribution, de plus en plus concentrée, exerce des pressions croissantes sur les entreprises situées en aval : elle demande (et fournit aussi) de meilleurs services pour les consommateurs. De nombreuses marques collectives peuvent être confrontées à des choix difficiles, par exemple si elles doivent livrer pour la marque (privée) du distributeur. Est-il intéressant de saisir ces nouvelles opportunités de marché ? Cela peut dépendre de l'effet global sur la perception de la marque collective par le consommateur, c'est-à-dire de l'effet sur la réputation collective. 
Il n'existe pas de réponses simples, ni de recette unique pour un comportement optimal ou une réaction optimale à l'évolution du marché et des besoins des distributeurs. On doit tenir compte de différents aspects et, bien souvent, plusieurs outils sont disponibles. Il ressort de l'analyse de divers cas que certaines caractéristiques communes ne sont pas prises en considération ou suffisamment explorées dans les modèles économiques existants. Il faudrait s'attacher à définir la configuration optimale d'une marque collective, de manière à faire concorder des incitations divergentes et à parvenir à un compromis efficient et équitable entre les divers acteurs des chaînes de distribution. Les problèmes sont plus complexes en présence d'entreprises hétérogènes, mais il existe de bonnes pratiques et, avec les leçons tirées des mauvaises expériences, elles peuvent permettre au secteur et aux pouvoirs publics de faire des choix éclairés.

Si ces problèmes revêtent une grande importance pour l'industrie agroalimentaire, ils intéressent également les économistes. Cet article a suggéré quelques thématiques pour les travaux de recherche à venir, afin de comprendre les équilibres dans le secteur et sur le marché tout en encourageant des décisions plus éclairées.
À ce jour, les pouvoirs publics ont pris acte de ces efforts collectifs dans le secteur agricole. Une bonne réglementation peut protéger les institutions ou les organisations utiles, mais elle ne peut les isoler de l'évolution du monde. Pour éviter d'être accusés de protéger indûment certaines entreprises, certains secteurs ou certaines régions du monde ${ }^{24}$, sans aucune justification commune et énoncée dans un accord, il faudrait que les économistes aident à déterminer comment la réputation collective pourrait contribuer à une offre de grande qualité et à l'amélioration des revenus des producteurs, mais à des prix raisonnables pour les consommateurs. À notre avis, les outils de l'économie de la réputation peuvent permettre d'explorer ces questions et des questions connexes.

L'auteur remercie F. Bergès, L. Maestri, S. Monier (quia réviséle texte) et(en particulier) Z. BouamraMechemache pour leur précieuse contribution. La clause d'exclusion de responsabilité habituelle s'applique. L'auteur a rédigé cet article alors qu'il était en visite à l'École d'économie de Toulouse, et exprime toute sa gratitude à cet établissement pour son hospitalité. Les recherches qui ont conduit au présent article ont bénéficié d'un financement du septième programme-cadre de I'Union européenne (FP7/2007-2013) au titre du financement no 331724 (ProdOrg\&FoodSupply).

24. Cf. notamment les récentes controverses suscitées par les négociations en cours sur le Partenariat transatlantique de commerce et d'investissement (The Economist, 2014). 


\section{RÉFÉRENCES BIBLIOGRAPHIQUES}

Akerlof G. A. (1970). The market for lemons: quality uncertainty and the market mechanism. Quarterly Journal of Economics, $\mathrm{n}^{\circ}$ 84, pp. 488-500.

ANF (1998). Compte rendu, $n^{\circ} 49$, Assemblée Nationale. Commission de la Production et des échanges.

Bar-Isaac H., Tadelis S. (2008). Seller reputation. Foundations and Trends in Microeconomics, vol. ${ }^{\circ}$, $\mathrm{n}^{\circ} 4$, pp. 273-351.

Barriquand N. (2008). Thiers et Laguiole à couteaux tirés. L'Express, July 3rd. http://www. lexpress.fr/region/thiers-et-laguiole-a-couteaux-tires_532495.html

Bertozzi L. (1995). Designation of origin: quality and specification. Food Quality and Preference $\mathrm{n}^{\circ}$ 6, pp. 143-147.

Bontemps C., Bouamra-Mechemache Z., Simioni M. (2013). Quality labels and firm survival: some first empirical evidence. European Review of Agricultural Economics, vol. 40, n 3, pp. 413-439.

Bureau J.-C., Valceschini E. (2003). European food-labeling policy: Successes and limitations. Journal of Food Distribution Research, vol. 34, n 3 , pp. 70-76.

Castriota S., Delmastro M. (2010). Individual and collective reputation: lessons from the wine market. L'Industria, vol. $31, \mathrm{n}^{\circ} 1$, pp. 149-172.

Costanigro M., Bond C. A., McCluskey J. J. (2012). Reputation leaders, quality laggards: Incentive structure in markets with both private and collective reputations. Journal of Agricultural Economics, vol. 63, n ${ }^{\circ}$, pp. 245-264.

Costanigro M., McCluskey J. J., Goemans C. (2010). The economics of nested names: name specificity, reputations, and price premia. American Journal of Agricultural Economics, vol. 92, $\mathrm{n}^{\circ}$ 5, pp. 1339-1350.

Costanzo E. (2013). L'Amarone da terreni pianeggianti. L'Arena, May 11th.

Crespi, John M., Marette S. (2001). How should food safety certification be financed? American Journal of Agricultural Economics, vol. 83, n 4, pp. 852-861.
Dell'Orefice G. (2013a). Accordini invita ad abbassare i toni: per l'Amarone non c'è alcun rischio-inflazione. Il Sole 24 Ore, May 9th.

Dell'Orefice G. (2013b). Vino, nuove regole per l'Amarone: l'area di produzione si estende in pianura. Il Sole 24 Ore, May 12th.

Dépêche (La) (2014). Laguiole encore privé de son nom et de la renommée de son savoirfaire. April 15th., http://www.ladepeche.fr/ article/2014/04/15/1863999-laguiole-encore-prive-nom-renommee-savoir-faire.html

Dielhenn M. (2014). L'Auvergne, la guerre des couteaux. Géo Arte, August 9th. http://www. arte.tv (seen on Arte-TV).

Economist (The) (2014). Food names. Stressed are the cheesemakers. July 19 th.

Fishman A., Finkelshtain I., Simhon A., Yacouel N. (2008). The economics of collective brands. Discussion paper $n^{\circ} 14 / 08$, The Center for Agricultural Economic Research, The Hebrew University of Jerusalem. Forthcoming in the Journal of Industrial Economics.

Fleckinger P. (2007). Collective reputation and market structure: Regulating the quality vs quantity trade-off. Cahier n ${ }^{\circ}$ 2007-26, École Polytechnique, CNRS.

Guerrini D. (2013a). Amarone, continua lo scontro tra Consorzio e Famiglie. Corriere di Verona - Corriere della Sera, May 12th.

Guerrini D. (2013b). Il Consorzio: Amarone, nessuna modifica lassista. Allegrini: è un condono. Corriere di Verona - Corriere della Sera, May 16th.

INA (1997). Polémique sur les Camemberts AOC sous la marque Leclerc. Institut National de l'Audio-visuel, January $30^{\text {th }}$, http://www.ina.fr/video/CNC9703054931/ polemique-sur-les-camemberts-aoc-sous-lamarque-leclerc-video.html.

Jefford A. (2008). Everything you always wanted to know about the Champagne area revisions but were afraid to ask. Andrew Jefford's Blog, July $11^{\text {th }}$, http://www.andrew jefford.com/node/134.

Johnson H. Robinson J. (2001). World atlas of wine. London, Octopus publishing group. 
Klein B., Leffler K. B. (1981). The role of market forces in assuring contractual performance. Journal of Political Economy, vol. 89, $\mathrm{n}^{\circ}$ 4, pp. 615-641.

Krebiehl A. (2013). Doubts surface over new Chianti classification. Decanter, March 8th. http://www.decanter.com/news/winenews/583702/doubts-surface-over-newchianti-classification.

Landon S., Smith C. E. (1998). Quality expectations, reputation and price. Southern Economic Journal, vol. 64, n 3, pp. 628-647.

Le Du G. (2014). Le lait AOP à la croisée des chemins. Ouest-France, June 12th., http:// www.ouest-france.fr/le-lait-aop-la-croiseedes-chemins-2620866.

Lewin M. W. (2009). The 1855 classification: on the mark or marketing ploy? Decanter, December 7th., http://www.decanter.com/ people-and-places/wine-articles/484012/ the-1855-classification-on-the-mark-ormarketing-ploy.

Lewin M. W. (2010). The classification system in Burgundy. Decanter, June 10th. http:// www.decanter.com/people-and-places/ wine-articles/483390/the-classificationsystem-in-burgundy.

Mailath G. J., Samuelson L. (2006). Repeated games and reputation,. New York, Oxford University Press.

Mailath G. J., Samuelson L. (2013). Reputations in repeated games. Handbook of Game Theory, vol. IV, New York, Elsevier.

Menapace L., G.C. Moschini (2012). Quality certification by geographical indications, trademarks and firm reputation. European Review of Agricultural Economics, vol. 39, $\mathrm{n}^{\circ} 4$, pp. 539-566.

Nelson P. (1970). Information and consumer behavior. Journal of Political Economy, vol. 78, n 2 , pp. 311-329.

Parisien (Le) (2013) Les couteliers de Thiers, défenseurs du Made in France bien avant l'heure. May 24th, http://www.leparisien.fr/ flash-actualite-economie/les-couteliers-dethiers-defenseurs-du-made-in-france-bienavant-1-heure-24-05-2013-2831055.php.

Rand M. (2013). Producer profile. Masi. Decanter, August, pp. 36-39.

Rinaldi M. (2012). Denominazione di Origine Cannubi Garantita. Il Tar del Lazio sconfessa Marchesi di Barolo. Intravino, June $20^{\text {th }}$, http://www.intravino.com/primo-piano/ denominazione-di-origine-cannubi-garantita-il-tar-del-lazio-sconfessa-marchesi-dibarolo/

Rinaldi M. (2013). La sentenza definitiva del Consiglio di Stato sulla collina dei Cannubi a Barolo farà discutere. Intravino, October $8^{\text {th }}$, http://www.intravino.com/grande-notizia/ la-sentenza-definitiva-del-consiglio-distato-sulla-collina-dei-cannubi-a-barolofara-discutere/

Fishman R. A. (2005). Is bigger better? Customer base expansion through word-of-mouth reputation. Journal of Political Economy, vol. $113, \mathrm{n}^{\circ} 5$, pp. 1147-1162.

Saak A. E. (2012). Collective reputation, social norms, and participation. American Journal of Agricultural Economics, vol. 94, $\mathrm{n}^{\circ} 3$, pp. 763-784.

Shapiro C. (1983). Premiums for high quality products as returns to reputations. Quarterly Journal of Economics, vol. 98, $\mathrm{n}^{\circ} 4$, pp. 659-680.

Suckling J. (2013). Cannubi Cannubi - More controversy? James Suckling's My Blog, October 14th. http://www.jamessuckling. com/cannubi-cannubi.html.

Tirole J. (1996). A theory of collective reputations (with applications to the persistence of corruption and to firm quality). Review of Economic Studies, $\mathrm{n}^{\circ}$ 63, pp. 1-22.

Winfree J. A., McCluskey J. J. (2005). Collective reputation and quality. American Journal of Agricultural Economics, n ${ }^{\circ}$ 87, pp. 206-213. 